\title{
Elementary operators on Banach algebras and Fourier transform
}

\author{
by \\ Miloš Arsenović and Dragoluub KečKić (Beograd)
}

\begin{abstract}
We consider elementary operators $x \mapsto \sum_{j=1}^{n} a_{j} x b_{j}$, acting on a unital Banach algebra, where $a_{j}$ and $b_{j}$ are separately commuting families of generalized scalar elements. We give an ascent estimate and a lower bound estimate for such an operator. Additionally, we give a weak variant of the Fuglede-Putnam theorem for an elementary operator with strongly commuting families $\left\{a_{j}\right\}$ and $\left\{b_{j}\right\}$, i.e. $a_{j}=a_{j}^{\prime}+i a_{j}^{\prime \prime}\left(b_{j}=b_{j}^{\prime}+i b_{j}^{\prime \prime}\right)$, where all $a_{j}^{\prime}$ and $a_{j}^{\prime \prime}\left(b_{j}^{\prime}\right.$ and $\left.b_{j}^{\prime \prime}\right)$ commute. The main tool is an $L^{1}$ estimate of the Fourier transform of a certain class of $C_{\mathrm{cpt}}^{\infty}$ functions on $\mathbb{R}^{2 n}$.
\end{abstract}

0. Introduction. The theory of generalized scalar operators on a Banach space was developed in [6]. Briefly, $a \in \mathcal{A}$ is a generalized scalar element of a unital Banach algebra $\mathcal{A}$ if it has real spectrum, and if for all real $t$, $\left\|e^{i t a}\right\| \leq C\left(1+|t|^{s}\right)$, for some constant $C$ depending only on $a$. Also, it is known that these two conditions are equivalent to the existence of a functional calculus for $a$, based on $\mathbb{R}$. If $s=0$, we say that such an element is pre-hermitian. In that case the condition of having real spectrum is not necessary. Also we can define pre-normal elements as elements of the form $h+i k$ with $h, k$ pre-hermitian. Many properties of pre-hermitian, pre-normal, and generalized scalar elements can be found in [6] and [5]. In Section 1 we review results concerning such elements, necessary for reading this note.

In [13], a functional calculus for several commuting operators on a Banach space, using Fourier transform, was developed. In Section 2, we prove two results about $L^{1}$ behaviour of the Fourier transforms of a family of $C_{\mathrm{cpt}}^{\infty}$ functions. These results have a central role in further applications to the theory of elementary operators on a unital Banach algebra.

Section 3 contains applications of the results from Section 2 to elementary operators on a unital Banach algebra $\mathcal{A}$, i.e. to mappings $\Lambda: \mathcal{A} \rightarrow \mathcal{A}$ of the form

2000 Mathematics Subject Classification: 47B48, 47B47, 42B10.

Key words and phrases: Banach algebras, elementary operators, Fuglede-Putnam theorem, Fourier transform. 


$$
\Lambda(x)=\sum_{j=1}^{n} a_{j} x b_{j} .
$$

These operators were introduced by Lumer and Rosenblum [11]. They have been investigated in many papers, first on the algebra $B(H)$ of all bounded operators on a separable Hilbert space $H$. For important results on elementary operators acting on a Banach algebra, or on the algebra of all bounded operators on a Banach space, the reader is referred to [12], [17], [18] and references therein.

We give three independent applications. The first of them is an ascent estimate for an elementary operator (1), with generalized scalar $a_{j}$ and $b_{j}$. For a linear mapping $\Lambda: E \rightarrow E$ on an arbitrary linear space $E$, the ascent $\operatorname{asc}(\Lambda)$ is defined as the least positive integer $k$ such that $\operatorname{ker}\left(\Lambda^{k}\right)=\operatorname{ker}\left(\Lambda^{k+1}\right)$. If no such positive integer exists we set $\operatorname{asc}(\Lambda)=+\infty$. We estimate the ascent of the operator (1) in terms of the orders of $a_{j}, b_{j}$ and the dimension of the set $\sigma\left(a_{1}, \ldots, a_{n}\right) \times \sigma\left(b_{1}, \ldots, b_{n}\right)$.

The second application is a weak variant of the Fuglede-Putnam theorem for the operator (1), where $\left\{a_{j}\right\}$ and $\left\{b_{j}\right\}$ are strongly commuting families. This means that $a_{j}=a_{j}^{\prime}+i a_{j}^{\prime \prime}, b_{j}=b_{j}^{\prime}+i b_{j}^{\prime \prime}$, where $\left\{a_{1}^{\prime}, a_{1}^{\prime \prime}, \ldots, a_{n}^{\prime}, a_{n}^{\prime \prime}\right\}$ and $\left\{b_{1}^{\prime}, b_{1}^{\prime \prime}, \ldots, b_{n}^{\prime}, b_{n}^{\prime \prime}\right\}$ are commuting families of generalized scalar elements. This weak Fuglede-Putnam theorem asserts that $\Lambda(x)=0$ implies $\left(\Lambda^{*}\right)^{k}(x)=0$ for some positive integer $k$, where $\Lambda^{*}(x)=\sum_{j=1}^{n} a_{j}^{*} x b_{j}^{*}$, and $a_{j}^{*}=a_{j}^{\prime}-i a_{j}^{\prime \prime}, b_{j}^{*}=b_{j}^{\prime}-i b_{j}^{\prime \prime}$. We determine $k$ in terms of the orders of $a_{j}^{\prime}$, $a_{j}^{\prime \prime}, b_{j}^{\prime}, b_{j}^{\prime \prime}$ and, once again, the dimension of the set $\sigma\left(a_{1}^{\prime}, a_{1}^{\prime \prime}, \ldots, a_{n}^{\prime}, a_{n}^{\prime \prime}\right) \times$ $\sigma\left(b_{1}^{\prime}, b_{1}^{\prime \prime}, \ldots, b_{n}^{\prime}, b_{n}^{\prime \prime}\right)$.

The third application is a norm estimate for the solution of the equation

$$
\sum_{j=1}^{n} a_{j} x b_{j}=y,
$$

in terms of the right hand side, provided that $0 \notin\left\{\lambda_{1} \mu_{1}+\cdots+\lambda_{n} \mu_{n} \mid \lambda_{j} \in\right.$ $\left.\sigma\left(a_{j}\right), \mu_{j} \in \sigma\left(b_{j}\right)\right\}$.

Finally, we conclude this note with some questions that we have not been able to answer.

\section{Preliminaries}

DeFINITION 1.1.

(a) We say that an element $a \in \mathcal{A}$ is hermitian if $\left\|e^{i t a}\right\|=1$ for all real $t$. The set of all hermitian elements of the algebra $\mathcal{A}$ is denoted by $\mathcal{H}(\mathcal{A})$.

(b) We say that an element $a \in \mathcal{A}$ is pre-hermitian if there exists $M<\infty$ such that $\left\|e^{i t a}\right\| \leq M$ for all real $t$. The set of all pre-hermitian elements of $\mathcal{A}$ is denoted by $\mathcal{H}_{1}(\mathcal{A})$. 
(c) We say that an element $a \in \mathcal{A}$ is normal if $a=h+i k$ for some $h, k \in \mathcal{H}(\mathcal{A})$ such that $h k=k h$, and pre-normal if $a=h+i k$ for some $h, k \in \mathcal{H}_{1}(\mathcal{A})$ such that $h k=k h$.

(d) The numerical range of $a \in \mathcal{A}$ is the set

$$
W(a)=\left\{f(a) \mid f \in \mathcal{A}^{*},\|f\|=1, f(e)=1\right\} .
$$

Proposition 1.1.

(a) $W(a)$ is always a closed convex subset of $\mathbb{C}$, and $\sigma(a) \subseteq W(a)$, where $\sigma(a)$ is the spectrum of $a$.

(b) $a \in \mathcal{A}$ is hermitian if and only if $W(a) \subseteq \mathbb{R}$, if and only if

$$
\|1+i t a\|=1+o(t) \quad \text { as } \mathbb{R} \ni t \rightarrow 0 .
$$

(c) A real linear combination of two hermitian elements is always hermitian.

(d) For a finite family of mutually commuting pre-hermitian elements, there exists a norm on $\mathcal{A}$ equivalent to the original one, making all of them hermitian.

(e) If $a=h+i k$, where $h, k \in \mathcal{H}(\mathcal{A})$, then $h$ and $k$ are uniquely determined.

Proof. Statements (a), (b), (c) and (e) are Theorems 1.3, 1.6 and Lemmas 5.2, 5.4 and 5.7 of [5], whereas statement (d) follows easily from Lemma 1.7 of [5].

\section{Proposition 1.2.}

(a) Let $a=h+i k$ be a pre-normal element, where $h, k \in \mathcal{H}_{1}(\mathcal{A})$, and suppose ax $=x a$ for some $x \in \mathcal{A}$. Then $(h-i k) x=x(h-i k)$, $h x=x h$ and $k x=x k$.

(b) If $a=h+i k$ is a pre-normal element, $h, k \in \mathcal{H}_{1}(\mathcal{A})$, then $h$ and $k$ are uniquely determined.

Proof. (a) The proof of this part is essentially the same as Rosenblum's well known proof of the Fuglede-Putnam theorem. Nevertheless we shall give it. Set $a^{*}=h-i k$. From $a x=x a$, it is easy to obtain by induction $\bar{\lambda}^{n} a^{n} x=x \bar{\lambda}^{n} a^{n}$ for all $\lambda \in \mathbb{C}$, and consequently $e^{\bar{\lambda} a} x=x e^{\bar{\lambda} a}$. Since $h k=k h$, it follows that $a a^{*}=a^{*} a$, and hence $e^{-\lambda a^{*}} x e^{\lambda a^{*}}=e^{\bar{\lambda} a-\lambda a^{*}} x e^{-\bar{\lambda} a+\lambda a^{*}}$. If we take $\lambda=\alpha+i \beta$, then we can easily compute $\bar{\lambda} a-\lambda a^{*}=2 i(\alpha k-\beta h)$, and also $e^{\bar{\lambda} a-\lambda a^{*}}=e^{i 2 \alpha k} e^{-i 2 \beta h}$ since $k$ and $h$ commute with each other. Therefore $\left\|e^{\bar{\lambda} a-\lambda a^{*}}\right\| \leq\left\|e^{i 2 \alpha k}\right\|\left\|e^{-i 2 \beta h}\right\| \leq M$. Now, the entire function $\lambda \mapsto e^{-\lambda a^{*}} x e^{\lambda a^{*}}=\varphi(\lambda)$ is bounded, and according to Liouville's theorem it is constant. Thus, $e^{-\lambda a^{*}} x e^{\lambda a^{*}}=\varphi(\lambda)=\varphi(0)=x$, i.e. $x e^{\lambda a^{*}}=e^{\lambda a^{*}} x$. Expanding both sides of this equation in a series, and comparing the coefficients, we get

$$
a^{*} x=x a^{*} .
$$


Adding (or subtracting) the initial equality we get the second and third equalities of the statement.

(b) Let $a=h+i k=h_{1}+i k_{1}$, where $h, h_{1}, k, k_{1}$ are pre-hermitian elements such that $h k=k h$ and $h_{1} k_{1}=k_{1} h_{1}$. Obviously, $a$ commutes with $a$, and by the previous part of this proposition, we conclude that all $h, k, h_{1}, k_{1}$ mutually commute. Now, by Proposition 1.1(d) there exists a norm, equivalent to the initial one, such that $h, h_{1}, k, k_{1}$ are all hermitian. Now, we have $h=h_{1}, k=k_{1}$.

The previous proposition allows us to define, for an arbitrary pre-normal $a=h+i k \in \mathcal{A}$, its adjoint $a^{*}=h-i k$.

Recall that from Vidav Palmer's well known theorem, $\mathcal{A}=\mathcal{H}(\mathcal{A})+i \mathcal{H}(\mathcal{A})$ if and only if $\mathcal{A}$ is a $C^{*}$-algebra.

Let $a \in \mathcal{A}$, and let $L_{a}, R_{a}: \mathcal{A} \rightarrow \mathcal{A}$ be given by $L_{a}(x)=a x$ and $R_{a}(x)=x a$. The following proposition carries over some of the properties of $a$ to the operators $L_{a}, R_{a} \in B(\mathcal{A})$.

Proposition 1.3.

(a) The mappings $a \mapsto L_{a}$ and $a \mapsto R_{a}$ are isometries and monomorphisms from the algebra $\mathcal{A}$ to the algebra $B(\mathcal{A})$.

(b) The spectra $\sigma\left(L_{a}\right)$ and $\sigma\left(R_{a}\right)$ coincide with $\sigma(a)$.

(c) $W\left(L_{a}\right)=W\left(R_{a}\right)=W(a)$.

(d) If a is (pre-)hermitian, then so are both $L_{a}$ and $R_{a}$.

(e) If $a=h+i k$ is (pre-)normal, then so are both $L_{a}=L_{h}+i L_{k}$ and $R_{a}=R_{h}+i R_{k}$.

We leave an easy proof to the reader.

Definition 1.2. We say that $a \in \mathcal{A}$ is a generalized scalar element if $e^{i t a}$ has polynomial growth for real $t$, i.e. there are constants $C, s$ such that

$$
\left\|e^{i t a}\right\| \leq C\left(1+|t|^{s}\right),
$$

and the spectrum of $a$ is real. In this case we say that $a$ has order $s$.

It is clear that every pre-hermitian element $a$ is a generalized scalar element of order 0, i.e. (2) holds with $s=0$. Also, there exists a norm equivalent to the initial one which makes $a$ hermitian. Changing norm does not change the spectrum. Thus $a$ has real spectrum.

In [7], for any $s>0$, an example is given of an element $S$ such that $\left\|e^{i t S}\right\| \approx|t|^{s}$ as $t \rightarrow \infty$.

2. Fourier transform. The basic tool we use to derive our results is a functional calculus for commuting families of generalized scalar operators, developed in [13]. 
Definition 2.1. $\check{L}_{1}^{s}=\check{L}_{1}^{s}\left(\mathbb{R}^{n}\right)$ is the set of all inverse Fourier transforms of functions from $\left\{g: \mathbb{R}^{n} \rightarrow \mathbb{C} \mid(1+|\xi|)^{s} g(\xi) \in L^{1}\left(\mathbb{R}^{n}\right)\right\}$.

In fact, $\check{L}_{1}^{s}$ is an algebra with respect to pointwise multiplication.

TheOREM 2.1. Let $S_{1}, \ldots, S_{n}$ be a commuting family of generalized scalar operators acting on a Banach space $X$, and let $s_{1}, \ldots, s_{n}$ be their orders. Then there is an algebra homomorphism $\Phi: \check{L}_{1}^{s} \rightarrow L(X)\left(s=s_{1}+\cdots+s_{n}\right)$ given by

$$
\Phi(f)\left(=f\left(S_{1}, \ldots, S_{n}\right)\right)=\frac{1}{(2 \pi)^{n / 2}} \int_{\mathbb{R}^{n}} \widehat{f}\left(\xi_{1}, \ldots, \xi_{n}\right) e^{i\left(\xi_{1} S_{1}+\cdots+\xi_{n} S_{n}\right)} d \xi,
$$

where $\widehat{f}$ denotes the Fourier transform of $f$, i.e.

$$
\widehat{f}(x)=\frac{1}{(2 \pi)^{n / 2}} \int_{\mathbb{R}^{n}} f(y) e^{-i x y} d y .
$$

The homomorphism $\Phi$ has the following properties:

(i) The integral in $(3)$ converges since $(1+|\xi|)^{s} \widehat{f}(\xi) \in L^{1}\left(\mathbb{R}^{n}\right)$ and exists as a Bochner integral.

(ii) If $f \equiv 0$ on the joint Taylor spectrum $\sigma_{\mathrm{T}}\left(S_{1}, \ldots, S_{n}\right)$ then we have $f\left(S_{1}, \ldots, S_{n}\right)=0$, and consequently, if $f \equiv g$ on $\sigma_{\mathrm{T}}\left(S_{1}, \ldots, S_{n}\right)$ then $f\left(S_{1}, \ldots, S_{n}\right)=g\left(S_{1}, \ldots, S_{n}\right)$.

(iii) For $f$ analytic in a neighborhood of the joint spectrum, $f\left(S_{1}, \ldots, S_{n}\right)$ has its usual meaning, obtained by power series expansion of $f$.

REMARK 2.1. Although the integral (3) exists as a Bochner integral, for our applications the following property of the integral of a function $f: \mathbb{R}^{n} \rightarrow$ $L(X)$ suffices: $\varphi\left(\int_{\mathbb{R}^{n}} f d x\right)=\int_{\mathbb{R}^{n}} \varphi \circ f d x$ for all bounded linear functionals $\varphi \in L(X)^{*}$.

REMARK 2.2. In [16], it was proved that the Taylor and Harte spectra of a commuting family of generalized scalar elements coincide.

The elementary operator (1) can be expressed as

$$
\Lambda=Q\left(L_{a_{1}}, \ldots, L_{a_{n}}, R_{b_{1}}, \ldots, R_{b_{n}}\right),
$$

where $Q(x)=x_{1} x_{n+1}+x_{2} x_{n+2}+\cdots+x_{n} x_{2 n}$ is a quadratic form on $\mathbb{R}^{2 n}$. Our aim is to estimate $\left\|e^{i t \Lambda}\right\|$ by calculating $e^{i t \Lambda}$ as $e^{i t Q\left(L_{a_{1}}, \ldots, R_{b_{n}}\right)}$. Unfortunately, $e^{i t Q} \notin L^{1}\left(\mathbb{R}^{2 n}\right)$, so it is impossible to calculate its Fourier transform, as a function. However, we can multiply $Q$ by a suitable $C_{\mathrm{cpt}}^{\infty}$ function which is equal to 1 on the joint spectrum of the $2 n$-tuple $\left(L_{a_{1}}, \ldots, L_{a_{n}}, R_{b_{1}}, \ldots, R_{b_{n}}\right)$. This spectrum is a compact subset, and we shall derive our results in terms of its dimension.

Let $K \subseteq \mathbb{R}^{2 n}$ be an arbitrary compact set. Recall that $K$ is said to have Hausdorff dimension $c$ if there exists a positive constant $N>0$ such that for 
all $\delta>0$ there exists a finite decomposition $K=\bigsqcup_{j=1}^{m} \beta_{j}$ with the following properties: (i) $\max _{1 \leq j \leq m} \operatorname{diam}\left(\beta_{j}\right)<\delta$ and (ii) $\sum_{j=1}^{m}\left(\operatorname{diam}\left(\beta_{j}\right)\right)^{c} \leq N$. We need a somewhat stronger concept of Hausdorff dimension, described in the following definition.

\section{DEFINITION 2.2.}

(a) We say that a compact set $K$ has balanced Hausdorff dimension $c$ if there exist positive constants $N, P>0$ such that for all $\delta>0$ there exists a finite covering $K \subseteq \bigsqcup_{j=1}^{m} \beta_{j}\left(\beta_{i} \cap \beta_{j}=\emptyset\right.$ !) with the following properties: (i) $\delta / P<\operatorname{diam}\left(\beta_{j}\right)<\delta$ for all $1 \leq j \leq m$ and (ii) $\sum_{j=1}^{m}\left(\operatorname{diam}\left(\beta_{j}\right)\right)^{c} \leq N$.

(b) We say that a function $f$ generates $e^{i t Q}$ on $K$ if $f \equiv e^{i t Q}$ on $K, f$ is analytic in a neighborhood of $K$, and $f \in C_{\mathrm{cpt}}^{\infty}$. The set of all such functions is denoted by $C_{Q}(K)$.

REMARK 2.3. One can verify that any subset of $\mathbb{R}^{2 n} C^{1}$-diffeomorphic to a $c$-dimensional simplex has balanced Hausdorff dimension $c$. In particular, every c-dimensional compact manifold, with or without boundary, has balanced Hausdorff dimension $c$.

Lemma 2.2. Let $K \subseteq \mathbb{R}^{2 n}$ be a set of balanced Hausdorff dimension c. Then for all $\delta>0$ there exists an open set $U_{\delta} \supset K$ such that $m\left(U_{\delta}\right) \leq$ $C(K, n) \delta^{2 n-c}$ and $\operatorname{dist}\left(K, U_{\delta}^{\mathrm{C}}\right) \geq \delta / P$.

Proof. Given $\delta>0$, let $K=\bigsqcup_{j=1}^{m} \beta_{j}$ be a decomposition of $K$ with properties (i) and (ii) from Definition 2.2(a). Set

$$
U_{\delta, j}=\left\{x \in \mathbb{R}^{2 n} \mid \operatorname{dist}\left(x, \beta_{j}\right)<d_{j}=\operatorname{diam}\left(\beta_{j}\right)\right\},
$$

and $U_{\delta}=\bigcup_{j=1}^{m} U_{\delta, j}$. Clearly, $\operatorname{dist}\left(K, U_{\delta}^{\mathrm{C}}\right) \geq \min d_{j} \geq \delta / P$. Also

$$
m\left(U_{\delta}\right) \leq \sum_{j=1}^{m} m\left(U_{\delta, j}\right) \leq\left|B_{2 n}\right| \sum_{j=1}^{m}\left(2 d_{j}\right)^{2 n}
$$

since $U_{\delta, j}$ is contained in some ball of radius $2 d_{j}$. (Here $\left|B_{2 n}\right|$ denotes the measure of the unit ball in $\mathbb{R}^{2 n}$.) Now, we have

$$
m\left(U_{\delta}\right) \leq C \sum_{j=1}^{m} d_{j}^{2 n}=C \sum_{j=1}^{m} d_{j}^{c} d_{j}^{2 n-c} \leq C \delta^{2 n-c} \sum_{j=1}^{m} d_{j}^{c} \leq C N \delta^{2 n-c} .
$$

Lemma 2.3. Let $K \subseteq \mathbb{R}^{2 n}$ be a compact set of balanced Hausdorff dimension $c$.

(a) For large $t$ there exists an open set $U_{t} \supset K$ such that $m\left(U_{t}\right) \leq$ $C(K, n) / t^{2 n-c}$ and $\operatorname{dist}\left(K, U_{t}^{\mathrm{C}}\right) \geq 1 / P t$. 
(b) There exists a $C^{\infty}$ function $\psi_{t}: \mathbb{R}^{2 n} \rightarrow \mathbb{R}$, analytic in a neighborhood of $K$, such that $0 \leq \psi_{t}(x) \leq 1$, and

$$
\psi_{t}(x)= \begin{cases}1, & x \in K, \\ 0, & x \notin U_{t},\end{cases}
$$

and $\left|\partial^{\alpha} \psi_{t} / \partial x^{\alpha}\right| \leq C_{\alpha} t^{|\alpha|}$ for any multiindex $\alpha$.

(c) For all positive integers $k$ we have

$$
\left|\Delta^{k}\left(e^{i t Q(x)} \psi_{t}(x)\right)\right|=O\left(t^{2 k}\right) \quad \text { as } t \rightarrow \infty .
$$

Proof. (a) Put $\delta=1 / t$ in Lemma 2.2.

(b) This part is in fact Proposition 1.3.5 from [2].

(c) Indeed, $\Delta^{k}$ is a differential operator of order $2 k$, so $\Delta^{k}\left(e^{i t Q(x)} \psi_{t}(x)\right)$ is a finite sum of terms, each containing a partial derivative of $\psi_{t}(x)$ of order $i$ and a partial derivative of $e^{i t Q(x)}$ of order $j$, with $i+j \leq 2 k$, and the result follows by parts (a) and (b).

TheOREM 2.4. Let $K \subseteq \mathbb{R}^{2 n}$ be a compact set of balanced Hausdorff dimension $c$. There exists a family of functions $\varphi_{t} \in C_{Q}(K)$ such that for any $\varepsilon>0$ the following estimate holds:

$$
\left\|\left(1+|\xi|^{s}\right) \widehat{\varphi}_{t}(\xi)\right\|_{1}=o\left(t^{s+c / 2+\varepsilon}\right) \quad(t \rightarrow \infty) .
$$

Proof. Set $\varphi_{t}(x)=\psi_{t}(x) e^{i t Q(x)}$, where $\psi_{t}$ are the functions from Lemma 2.3(b). For large $|x|$, using Lemma 2.3(c), we have

$$
\begin{aligned}
\left|\left(1+|x|^{s}\right) \widehat{\varphi}_{t}(x)\right| & =\left(1+|x|^{s}\right) \frac{1}{(2 \pi)^{n}|x|^{2 k}}\left|\int_{\mathbb{R}^{2 n}} \varphi_{t}(\xi) \Delta^{k} e^{-i x \xi} d \xi\right| \\
& =\left(1+|x|^{s}\right) \frac{1}{(2 \pi)^{n}|x|^{2 k}}\left|\int_{\mathbb{R}^{2 n}} \Delta^{k} \varphi_{t}(\xi) e^{-i x \xi} d \xi\right| \\
& \leq C_{1}^{\prime} \frac{t^{2 k} m\left(U_{t}\right)}{|x|^{2 k-s}} \leq C_{1} \frac{t^{2 k-2 n+c}}{|x|^{2 k-s}} .
\end{aligned}
$$

Now, using the Cauchy-Schwarz inequality and Plancherel's theorem we get

$$
\begin{aligned}
\left\|\left(1+|x|^{s}\right) \widehat{\varphi}_{t}(x)\right\|_{1} \\
\quad=\int_{|x| \leq M}\left|\left(1+|x|^{s}\right) \widehat{\varphi}_{t}(x)\right| d x+\int_{|x| \geq M}\left(1+|x|^{s}\right)\left|\widehat{\varphi}_{t}(x)\right| d x \\
\quad \leq\left(\int_{|x| \leq M}\left(1+|x|^{s}\right)^{2} d x\right)^{1 / 2}\left\|\widehat{\varphi}_{t}\right\|_{2}+C_{1} t^{2 k-2 n+c} \int_{|x| \geq M} 1 /|x|^{2 k-s} d x
\end{aligned}
$$




$$
\begin{aligned}
& \leq K_{1}^{\prime} M^{s} m\{|x| \leq M\}^{1 / 2}\left\|\widehat{\varphi}_{t}\right\|_{2}+C_{1} t^{2 k-2 n+c} \int_{|x| \geq M} 1 /|x|^{2 k-s} d x \\
& \leq K_{1} M^{n+s} t^{c / 2-n}+K_{2} t^{2 k-2 n+c} M^{2 n-2 k+s} .
\end{aligned}
$$

If we put $M=t^{1+\frac{c / 2}{2 k-n}}$ we get $\left\|\widehat{\varphi}_{t}\right\|_{1} \leq K t^{\frac{(2 s+c) k-s(n-c / 2)}{2 k-n}}$. As $k$ can be arbitrarily large this proves (4).

Next, we want to improve estimate (4) under additional assumptions.

THEOREM 2.5. Let $K$ be a subset of a c-dimensional affine subspace $x_{0}+V \subseteq \mathbb{R}^{2 n}$, where $V$ is a vector subspace of $\mathbb{R}^{2 n}$, and $0 \leq c \leq 2 n$ is an integer. Assume $Q$ is nondegenerate on $V$. There exists a family of functions $\varphi_{t} \in C_{Q}(K)$ such that

$$
\left\|\widehat{\varphi}_{t}(\xi)\right\|_{1}=O\left(t^{c / 2}\right) \quad(t \rightarrow \infty) .
$$

Proof. First, we can assume $V$ is a linear subspace of $\mathbb{R}^{2 n}$; next we choose a basis in $V$ such that in the new coordinates $Q(x)=\sum_{j=1}^{c} \lambda_{j} y_{j}^{2}(x \in V)$, where $\lambda_{j} \in\{1,-1\}$. This basis can be extended, using Witt's theorem [10, XIV.5], to a basis of $\mathbb{R}^{2 n}$ such that, in the new coordinates,

$$
Q(x)=\sum_{j=1}^{2 n} \lambda_{j} y_{j}^{2}, \quad \lambda_{j} \in\{1,-1\} .
$$

We have here a linear change of variables $x=B y$. Choose $R>0$ such that $\left|y_{j}\right| \leq R$ for all points in $K$ and define $\varphi_{t}(x)=\psi_{t}(x) \exp (i t Q(x))$, where

$$
\psi_{t}(x)=\prod_{p=1}^{c} g\left(y_{p}\right) \prod_{q=1}^{2 n-c} f\left(\sqrt{t} y_{c+q}\right)=\chi_{t}(y)
$$

and $f, g \in C_{\mathrm{cpt}}^{\infty}(\mathbb{R})$ are such that $f=1$ in a neighborhood of $0, g(y)=1$ if $-R \leq y \leq R$, and $g(y)=0$ for $y \geq 2 R$. We have

$$
\begin{aligned}
\widehat{\varphi}_{t}(\xi) & =\frac{1}{(2 \pi)^{n}} \int_{\mathbb{R}^{2 n}} \psi_{t}(x) \exp (i t Q(x)) e^{-i x \cdot \xi} d x \\
& =\frac{1}{(2 \pi)^{n}} \int_{\mathbb{R}^{2 n}} \chi_{t}(y) e^{i t Q(B y)} e^{-i B y \cdot \xi} d B y \\
& =\frac{|\operatorname{det} B|}{(2 \pi)^{n}} \int_{\mathbb{R}^{2 n}} \chi_{t}(y) \exp \left(i t \sum \lambda_{j} y_{j}^{2}\right) e^{-i y \cdot B^{t} \xi} d y \\
& =|\operatorname{det} B| \widehat{\theta}_{t}\left(B^{t} \xi\right),
\end{aligned}
$$

where

$$
\theta_{t}(y)=\chi_{t}(y) \exp \left(i t \sum \lambda_{j} y_{j}^{2}\right)=\prod_{p=1}^{c} u_{t}\left(y_{p}\right) \cdot \prod_{q=1}^{2 n-c} v_{t}\left(y_{q+c}\right),
$$

and $u_{t}(y)=e^{ \pm i t y^{2}} g(y), v_{t}(y)=e^{ \pm i t y^{2}} f(\sqrt{t} y)$. 
Since $\left\|\widehat{v}_{t}\right\|_{L^{1}(\mathbb{R})}$ does not depend on $t$ we use Fubini's theorem to reduce (5) to the following

LEMma 2.6. $\left\|\widehat{u}_{t}\right\|_{L^{1}(\mathbb{R})}=O(\sqrt{t})$ as $t \rightarrow \infty$.

This lemma follows easily from the following estimates:

$1^{\circ}\left|\widehat{u}_{t}(\eta)\right| \leq C / \sqrt{t}$ for $\eta \in[-6 R t, 6 R t]$, where $C$ does not depend on $t$ and $\eta$.

$2^{\circ}\left|\widehat{u}_{t}(\eta)\right| \leq C /|\eta|^{2}$ for $\eta \geq 6 R t$, where $C$ does not depend on $t$ and $\eta$.

Proof of $1^{\circ}$. We have

(6) $\widehat{u}_{t}(\eta)=\frac{e^{-i \eta^{2} / 4 t}}{(2 \pi)^{n}} \int_{-\infty}^{\infty} e^{i t(y-\eta / 2 t)^{2}} g(y) d y=\frac{e^{-i \eta^{2} / 4 t}}{(2 \pi)^{n}} \int_{-\infty}^{\infty} e^{i t z^{2}} g_{\alpha}(z) d z$

where $g_{\alpha}(z)=g(z+\alpha)$ and $\alpha=\eta / 2 t$. Note that $|\alpha| \leq 3 R$. Therefore $g_{\alpha}(z)$, $|\alpha| \leq 3 R$ is a bounded family of functions in the $C_{\mathrm{cpt}}^{\infty}(\mathbb{R})$ topology, so the stationary phase method [9] gives the following estimate, uniformly over $|\alpha| \leq 3 R$ :

$$
\int_{-\infty}^{\infty} e^{i t z^{2}} g_{\alpha}(z) d z=O(1 / \sqrt{t})
$$

We present here a proof for the reader's convenience. First, note that $g_{\alpha}(z)=g_{\alpha}(0)+z \gamma_{\alpha}(z)$, where $\gamma_{\alpha}(z)=\int_{0}^{1} g^{\prime}(z s+\alpha) d s$ and therefore

$$
\int_{-\infty}^{\infty} e^{i t z^{2}} g_{\alpha}(z) d z=\lim _{A \rightarrow \infty} \int_{-A}^{A} g_{\alpha}(0) e^{i t z^{2}} d z+\lim _{A \rightarrow \infty} \int_{-A}^{A} e^{i t z^{2}} z \gamma_{\alpha}(z) d z .
$$

The first limit is equal to $g_{\alpha}(0) e^{i \pi / 4} \sqrt{\pi / t}$, which is $O(1 / \sqrt{t})$ uniformly over $\alpha$. This also shows that the second limit exists. Next,

$$
\begin{aligned}
\lim _{A \rightarrow \infty} \int_{-A}^{A} e^{i t z^{2}} z \gamma_{\alpha}(z) d z & =\frac{1}{2 i t} \lim _{A \rightarrow \infty} \int_{-A}^{A} \gamma_{\alpha}(z) \frac{d}{d z} e^{i t z^{2}} d z \\
& =\frac{1}{2 i t} \lim _{A \rightarrow \infty}\left[\left.\gamma_{\alpha}(z) e^{i t z^{2}}\right|_{-A} ^{A}-\int_{-A}^{A} \gamma_{\alpha}^{\prime}(z) e^{i t z^{2}} d z\right] \\
& =\frac{-1}{2 i t} \lim _{A \rightarrow \infty} \int_{-A}^{A} \gamma_{\alpha}^{\prime}(z) e^{i t z^{2}} d z
\end{aligned}
$$

because $\gamma_{\alpha}(z)=\left(g_{\alpha}(z)-g_{\alpha}(0)\right) / z=o(1)$ as $|z| \rightarrow \infty$.

Next, note that

$$
\gamma_{\alpha}^{\prime}(z)=\frac{g_{\alpha}^{\prime}(z) z-g_{\alpha}(z)+g_{\alpha}(0)}{z^{2}}=\frac{g_{\alpha}(0)}{z^{2}} \quad \text { for }|z|>5 R,
$$


hence

$$
\begin{aligned}
\int_{-\infty}^{\infty}\left|\gamma_{\alpha}^{\prime}(z) e^{i t z^{2}}\right| d z & \leq 2\left|g_{\alpha}(0)\right| \int_{5 R}^{\infty} \frac{d z}{z^{2}}+\int_{-5 R}^{5 R}\left|\gamma_{\alpha}^{\prime}(z)\right| d z \\
& \leq \frac{2\left|g_{\alpha}(0)\right|}{5 R}+10 R \cdot \max _{\alpha}\left|\int_{0}^{1} g^{\prime \prime}(z s+\alpha) s d s\right|=C .
\end{aligned}
$$

This shows that $\lim _{A \rightarrow \infty} \int_{-A}^{A} \gamma_{\alpha}^{\prime}(z) e^{i t z^{2}} d z$ is uniformly bounded over $t \in \mathbb{R}$ and $|\alpha| \leq 3 R$. Hence ( 7$)$ gives

$$
\lim _{A \rightarrow \infty} \int_{-A}^{A} e^{i t z^{2}} z \gamma_{\alpha}(z) d z=O(1 / t),
$$

and $1^{\circ}$ is proved.

Proof of $2^{\circ}$. We can ignore the factor $\frac{e^{-i \eta^{2} / 4 t}}{(2 \pi)^{n}}$. We have

$$
\begin{aligned}
\int_{-\infty}^{\infty} e^{i t z^{2}} g_{\alpha}(z) d z & =\frac{1}{2 i t} \int_{-\infty}^{\infty} \frac{g_{\alpha}(z)}{z} \frac{d}{d z}\left(e^{i t z^{2}}\right) d z \\
& =-\frac{1}{2 i t} \int_{-\infty}^{\infty} e^{i t z^{2}}\left(\frac{g_{\alpha}(z)}{z}\right)^{\prime} d z \\
& =-\frac{1}{(2 i t)^{2}} \int_{-\infty}^{\infty}\left(e^{i t z^{2}}\right)^{\prime} \frac{1}{z}\left(\frac{g_{\alpha}(z)}{z}\right)^{\prime} d z \\
& =-\frac{1}{4 t^{2}} \int_{-\infty}^{\infty} e^{i t z^{2}} \frac{d}{d z}\left[\frac{g_{\alpha}^{\prime}(z)}{z^{2}}-\frac{g_{\alpha}(z)}{z^{3}}\right] d z
\end{aligned}
$$

and this is a sum of four terms of the form

$$
\pm \frac{1}{4 t^{2}} \int_{-\infty}^{\infty} e^{i t z^{2}} \frac{G(z+\alpha)}{z^{k}} d z,
$$

where $G$ stands for one of the functions $g, g^{\prime}$ or $g^{\prime \prime}$ and $k \in\{2,3,4\}$. Consider one such term; changing variables one gets

$$
\begin{aligned}
\frac{1}{4 t^{2}} \int_{-\infty}^{\infty} e^{i t(z-\alpha)^{2}} \frac{G(z)}{(z-\alpha)^{k}} d z & =\frac{e^{i t \alpha^{2}}}{4 t^{2}} \int e^{i t z^{2}} e^{-i \eta \cdot z} \frac{G(z)}{(z-\alpha)^{k}} d z \\
& =-e^{i t \alpha^{2}} \frac{1}{4 t^{2} \eta^{2}} \int e^{i t z^{2}} \frac{G(z)}{(z-\alpha)^{k}} \frac{d^{2}}{d z^{2}} e^{-i \eta z} d z \\
& =-\frac{e^{i t \alpha^{2}}}{4 t^{2} \eta^{2}} \int_{-2 R}^{2 R} e^{-i \eta z} \frac{d^{2}}{d z^{2}}\left[e^{i t z^{2}} \frac{G(z)}{(z-\alpha)^{k}}\right] d z
\end{aligned}
$$


Since $|\alpha| \geq 3 R$, we have a uniform estimate

$$
\left|\frac{d^{2}}{d z^{2}}\left[e^{i t z^{2}} \frac{G(z)}{(z-\alpha)^{k}}\right]\right| \leq C t^{2}
$$

for $|z| \leq 2 R$ and $|\alpha| \geq 3 R$, hence each of the four terms is estimated by $C / \eta^{2}$, as needed.

3. Elementary operators. Our first result is a simple consequence of results from the previous section.

THEOREM 3.1.

(a) Let $a_{1}, \ldots, a_{n}$, and $b_{1}, \ldots, b_{n}$ be commuting $n$-tuples of generalized scalar elements of a unital Banach algebra $\mathcal{A}$, with orders $s_{1}, \ldots, s_{n}$, and $r_{1}, \ldots, r_{n}$ respectively. Also, let $s=s_{1}+\cdots+s_{n}, r=r_{1}+\cdots+r_{n}$ be their total orders. Then the elementary operator $\Lambda$ given by (1) is also a generalized scalar operator. Its order is $r+s+c / 2+\varepsilon$ for any $\varepsilon>0$, where $c$ is the balanced Hausdorff dimension of the set $K=\sigma\left(a_{1}, \ldots, a_{n}\right) \times \sigma\left(b_{1}, \ldots, b_{n}\right)$, where $\sigma$ denotes the joint spectrum defined in [8].

(b) If, in addition, $s=r=0$ and $K$ is contained in an affine subspace of $\mathbb{R}^{2 n}$ of integer dimension $c$, then $\Lambda$ is a generalized scalar operator with order at most $c / 2$.

Proof. (a) From a result of Harte and Hernandez [8] it follows that

$$
\sigma\left(L_{a_{1}}, \ldots, L_{a_{n}}, R_{b_{1}}, \ldots, R_{b_{n}}\right) \subseteq \sigma\left(a_{1}, \ldots, a_{n}\right) \times \sigma\left(b_{1}, \ldots, b_{n}\right)=K .
$$

Also, using Proposition 1.3, it is easy to verify that the operators $L_{a_{1}}, \ldots, L_{a_{n}}$, $R_{b_{1}}, \ldots, R_{b_{n}}$ form a commuting family of generalized scalar operators on $\mathcal{A}$ considered as a Banach space. Take the functions $\varphi_{t}$ from Theorem 2.4. Since $\varphi_{t}=\exp (i t Q)$ on $K$, from Theorems 2.1 and 2.4 it follows that

$$
\|\exp (i t \Lambda)\|=\left\|\varphi_{t}(\Lambda)\right\| \leq\left\|\widehat{f}(\xi) \alpha\left(1+|\xi|^{s}\right)\right\|_{1}=o\left(t^{s+r+c / 2+\varepsilon}\right),
$$

where $c$ is the balanced dimension of $K$.

(b) The proof of the second part is the same. The only difference is that we apply Theorem 2.5 instead of Theorem 2.4.

In the worst case the dimension of $K$ might be $2 n$, so we get the following corollary.

Corollary 3.2. Let $\left\{a_{1}, \ldots, a_{n}\right\}$ and $\left\{b_{1}, \ldots, b_{n}\right\}$ be two $n$-tuples of commuting pre-hermitian elements of a unital Banach algebra $\mathcal{A}$. Then the operator $\Lambda: \mathcal{A} \rightarrow \mathcal{A}$ is a generalized scalar operator, and its order is at most n.

It seems, from $1^{\circ}$ in the proof of Lemma 2.6, that this estimate is the best one can obtain via Fourier transform. However, considering the following 
example one can conjecture that if $\mathcal{A}$ is a $C^{*}$-algebra a better estimate $\left\|e^{i t \Lambda}\right\|=O\left(t^{n / 2}\right)$ holds.

Example 3.1. Let $H=L^{2}(0,1)$, and let $A: H \rightarrow H$ be given by $A f(s)=s f(s)$. Consider the mapping $X \mapsto \Lambda(X)=A X A$ on $B(H)$. Note that $\Lambda: B(H) \rightarrow B(H)$ is the adjoint of the multiplication operator $M$ : $\mathfrak{S}_{1} \rightarrow \mathfrak{S}_{1}$ of the same form $M(X)=A X A$, where $\mathfrak{S}_{1}$ stands for the ideal of all nuclear operators. This can be used to reduce the norm estimate of $e^{i t \Lambda}$ to a norm estimate of $e^{i t M}$.

If $X$ is a nuclear operator, then it can be expressed as an integral operator with kernel $K, X f(s)=\int_{0}^{1} K(s, u) f(u) d u$. Straightforward calculation gives $M^{n}(X) f(s)=\int_{0}^{1} s^{n} K(s, u) u^{n} f(u) d u$, and

$$
e^{i t M}(X) f(s)=\left(\sum_{n=0}^{\infty} i^{n} t^{n} M^{n}(X) / n !\right) f(s)=\int_{0}^{1} e^{i t s u} K(s, u) f(u) d u .
$$

Thus $e^{i t M}$ is a Schur multiplier with symbol $e^{i t s u}$. From [4] it follows that its norm does not exceed

$$
C \underset{0<s<1}{\operatorname{ess} \sup }\left\|u \mapsto e^{i t s u}\right\|_{W_{2}^{\alpha}}
$$

for all $\alpha>1 / 2$, where $W_{2}^{\alpha}$ stands for the Sobolev space of index $\alpha$. It is easy to verify that the last expression is $O\left(t^{\alpha}\right)$.

REMARK 3.1. The estimate $\|\exp (i t \Lambda)\|=O\left(t^{s+r+2 n}\right)$ as $t \rightarrow \infty$ for $s, r$ integers follows from a paper by Albrecht [1]. If $s, r$ are half-integers then from [1] one can derive only $\|\exp (i t \Lambda)\|=O\left(t^{s+r+3 n}\right)$. Our estimate is a refinement of the last one.

Let $E$ be an arbitrary linear space, and let $T: E \rightarrow E$ be an arbitrary linear mapping. The ascent of $T$ is defined as the least integer $m$ such that $\operatorname{ker} T^{m+1}=\operatorname{ker} T^{m}$. The ascent of $T$ is usually denoted by $\operatorname{asc}(T)$. Clearly $\operatorname{asc} T=0$ if and only if $T$ is injective. Also $\operatorname{asc}(T) \leq 1$ if and only if $\operatorname{ker} T$ and $T(E)$ have trivial intersection. The finite ascent leads to the property of being semifredholm.

Theorem 3.3. Let $X$ be a Banach space, and let $S: X \rightarrow X$ be a generalized scalar operator of order $s$. Then the ascent of $S$ is finite, and $\operatorname{asc}(S) \leq[s]+1$.

Proof. Indeed, suppose that $S^{k+1}(x)=0$ for some $x \in X$, where $k>s$ is a positive integer. Then $e^{i t S}(x)=\sum_{j=0}^{k}(i t)^{j} S^{j}(x) / j$ ! , and also

$$
S^{k}(x)=k !\left(e^{i t S}(x)-\sum_{j=0}^{k-1}(i t)^{j} S^{j}(x) / j !\right) /(i t)^{k} .
$$


Since $S$ is a generalized scalar operator, we obtain

$$
\begin{aligned}
\left\|S^{k}(x)\right\| & \leq \frac{k !\left\|e^{i t S}\right\|\|x\|+\sum_{j=0}^{k-1} t^{j}\left\|S^{j}(x)\right\| / j !}{t^{k}} \\
& \leq \frac{k !\left(C t^{s}\|x\|+\sum_{j=0}^{k-1} t^{j}\left\|S^{j}(x)\right\| / j !\right)}{t^{k}} \rightarrow 0 \quad(t \rightarrow \infty),
\end{aligned}
$$

implying $S^{k}(x)=0$, as required.

Corollary 3.4. Let $\mathcal{A}$ be a unital Banach algebra, and let $\left\{a_{1}, \ldots\right.$, $\left.a_{n}\right\}$ and $\left\{b_{1}, \ldots, b_{n}\right\}$ be two n-tuples of commuting generalized scalar elements of $\mathcal{A}$, with orders $s_{1}, \ldots, s_{n}$ and $r_{1}, \ldots, r_{n}$, respectively. If $\Lambda: \mathcal{A} \rightarrow \mathcal{A}$ is an elementary operator given by $\Lambda(x)=\sum_{j=1}^{n} a_{j} x b_{j}$, then $\operatorname{asc}(\Lambda)<\infty$. Moreover, $\operatorname{asc}(\Lambda) \leq[s+r+c / 2]+1$, where $s=s_{1}+\cdots+s_{n}, r=r_{1}+$ $\cdots+r_{n}$, and $c$ is the balanced Hausdorff dimension of the set $\sigma\left(a_{1}, \ldots, a_{n}\right) \times$ $\sigma\left(b_{1}, \ldots, b_{n}\right)$.

Proof. It suffices to combine Theorems 3.1 and 3.3 .

REMARK 3.2. In [19] it was proved that asc $\Lambda \leq(2+8(s+r)) n-1$. The previous corollary, even in the worst case $c=2 n$, is a refinement of this result. Also, if $a_{j}, b_{j}$ are pre-hermitian elements with finite spectra we have $\operatorname{asc}(\Lambda) \leq 1$.

We say that the family $\left\{U_{1}, \ldots, U_{n}\right\}$ is strongly commuting if $U_{j}=$ $S_{j}+i T_{j}$, where $\left\{S_{1}, \ldots, S_{n}, T_{1}, \ldots, T_{n}\right\}$ is a commuting family of generalized scalar elements.

The following theorem is a variant of the classical Fuglede-Putnam theorem.

Theorem 3.5. Let $\mathcal{A}$ be a unital Banach algebra, let $a_{j}=a_{j}^{\prime}+i a_{j}^{\prime \prime}$, $b_{j}=b_{j}^{\prime}+i b_{j}^{\prime \prime} \in \mathcal{A}(1 \leq j \leq n)$ be two strongly commuting families, and let $s=\sum_{j=1}^{n}\left(s_{j}^{\prime}+s_{j}^{\prime \prime}\right)$ and $r=\sum_{j=1}^{n}\left(r_{j}^{\prime}+r_{j}^{\prime \prime}\right)$ be the total orders of the families $a_{j}$ and $b_{j}$. Define $\Lambda(x)=\sum a_{j} x b_{j}$ and $\Lambda^{*}(x)=\sum a_{j}^{*} x b_{j}^{*}\left(\Lambda, \Lambda^{*}: \mathcal{A} \rightarrow \mathcal{A}\right)$, where $a_{j}^{*}=a_{j}^{\prime}-i a_{j}^{\prime \prime}, b_{j}^{*}=b_{j}^{\prime}-i b_{j}^{\prime \prime}$. If $\Lambda(x)=0$, then $\left(\Lambda^{*}\right)^{k}(x)=0$ for some positive integer $k$. Further $k \leq[s+r+c / 2]+1$, where $c$ denotes the balanced Huadorff dimension of $\sigma\left(a_{1}^{\prime}, a_{1}^{\prime \prime}, \ldots, a_{n}^{\prime}, a_{n}^{\prime \prime}\right) \times \sigma\left(b_{1}^{\prime}, b_{1}^{\prime \prime}, \ldots, b_{n}^{\prime}, b_{n}^{\prime \prime}\right)$.

Proof. (a) It is clear that $\Lambda(x)=\Lambda_{1}(x)+i \Lambda_{2}(x)$ and $\Lambda^{*}(x)=\Lambda_{1}(x)-$ $i \Lambda_{2}(x)$, where

$$
\Lambda_{1}(x)=\sum\left(a_{j}^{\prime} x b_{j}^{\prime}-a_{j}^{\prime \prime} x b_{j}^{\prime \prime}\right), \quad \Lambda_{2}(x)=\sum\left(a_{j}^{\prime \prime} x b_{j}^{\prime}+a_{j}^{\prime} x b_{j}^{\prime \prime}\right) .
$$

It is also clear that $\Lambda_{1}$ and $\Lambda_{2}$ commute. From Theorem 3.1 we know that $\left\|\exp \left(i t \Lambda_{1}\right)\right\|,\left\|\exp \left(i t \Lambda_{2}\right)\right\|=O\left(t^{\mu}\right)$, where $\mu=s+r+c / 2+\varepsilon$ and $\varepsilon$ is sufficiently small. 
Suppose now that $\Lambda(x)=0$. We have $\Lambda_{1}(x)=-i \Lambda_{2}(x)$, and by induction $\Lambda_{1}^{n}(x)=\left(-i \Lambda_{2}\right)^{n}(x)$, and therefore $\exp \left(\Lambda_{1}\right)(x)=\exp \left(-i \Lambda_{2}\right)(x)$. Let $\lambda=$ $\alpha+i \beta \in \mathbb{C}$, and let $f$ be an arbitrary functional from $\mathcal{A}^{*}$, the dual space of $\mathcal{A}$ considered as a Banach space. We get

$$
\begin{aligned}
\left|f\left(\exp \left(\lambda \Lambda_{1}\right)(x)\right)\right| & =\left|f\left(\exp \left(i \beta \Lambda_{1}\right) \exp \left(\alpha \Lambda_{1}\right)(x)\right)\right| \\
& =\left|f\left(\exp \left(i \beta \Lambda_{1}\right) \exp \left(-i \alpha \Lambda_{2}\right)(x)\right)\right| \\
& \leq\|f\| C(\alpha \beta)^{\mu}\|x\| \leq\|f\| C_{1}|\lambda|^{2 \mu}\|x\| .
\end{aligned}
$$

Since $\lambda \mapsto f\left(\exp \left(\lambda \Lambda_{1}\right)(x)\right)$ is an entire function, from Cauchy's formulae for the coefficients in the power series expansion it follows that this function is a polynomial of degree at most $2 \mu$. Hence $f\left(\Lambda_{1}^{m}(x)\right)=0$ for all $f \in \mathcal{A}^{*}$ and $m>2 \mu$. Invoking the Hahn-Banach theorem we conclude that $\Lambda_{1}^{m}(x)=0$ for all $m>2 \mu$. By Corollary 3.4 the ascent of the operator $\Lambda_{1}$ does not exceed $k=[s+r+c / 2]+1$. Since $2 \mu>k$, it follows that $\Lambda_{1}^{k}(x)=0$. Also $\Lambda_{1}^{j} \Lambda_{2}^{k-j} x=i^{k-j} \Lambda_{1}^{k}(x)=0$, and therefore

$$
\left(\Lambda^{*}\right)^{k}(x)=\left(\Lambda_{1}-i \Lambda_{2}\right)^{k}(x)=\sum_{j=0}^{k}(-i)^{k-j} \Lambda_{1}^{j} \Lambda_{2}^{k-j}(x)=0 .
$$

REMARK 3.3. Note that for given $a_{j}=a_{j}^{\prime}+i a_{j}^{\prime \prime}$, where $a_{j}^{\prime}$ and $a_{j}^{\prime \prime}$ are commuting generalized scalar elements we do not claim that this representation is unique, so $a_{j}^{*}$ is not uniquely determined.

REMARK 3.4. The worst case is $c=4 n$ from which we get $k \leq[s+r$ $+2 n]+1$ in any case. The best case is where all $a_{j}$ and $b_{j}$ are pre-normal and $c=0$, for instance pre-normal elements with finite spectra. Then we can claim $k=1$, and that is the strong Fuglede-Putnam theorem.

Consider the equation $\sum_{j=1}^{n} a_{j} x b_{j}=y$. The problem of estimating the norm of $\|x\|$ in terms of $\|y\|$ is very well known. It amounts to estimating $\left\|\Lambda^{-1}\right\|$. See for instance [14] and [3]. In [14] it was proved that

$$
\|x\| \leq \frac{C}{\delta}\left(\frac{\max \{1, \delta\}}{\delta}\right)^{s}\|y\|
$$

where $s$ is the order of $\Lambda$ and where $\delta=\inf \left\{\left|\sum \lambda_{j} \mu_{j}\right| \mid\left(\lambda_{1}, \ldots, \lambda_{n}\right) \in\right.$ $\left.\sigma\left(a_{1}, \ldots, a_{n}\right),\left(\mu_{1}, \ldots, \mu_{n}\right) \in \sigma\left(b_{1}, \ldots, b_{n}\right)\right\}$. However, the existence of $s$ was only proved indirectly, and no exact value was given.

The following theorem gives this estimate with an explicit formula for $s$.

THEOREM 3.6.

(a) Let $\mathcal{A}$ be a unital Banach algebra, and let $a_{1}, \ldots, a_{n}$ and $b_{1}, \ldots, b_{n}$ be two n-tuples of commuting generalized scalar elements of $\mathcal{A}$, with orders $s_{1}, \ldots, s_{n}$ and $r_{1}, \ldots, r_{n}$, respectively. Also, let $\Lambda: \mathcal{A} \rightarrow \mathcal{A}$ be an elementary operator given by (1). If $0 \notin\left\{\lambda_{1} \mu_{1}+\cdots+\lambda_{n} \mu_{n} \mid\right.$ 
$\left.\left(\lambda_{1}, \ldots, \lambda_{n}\right) \in \sigma\left(a_{1}, \ldots, a_{n}\right),\left(\mu_{1}, \ldots, \mu_{n}\right) \in \sigma\left(b_{1}, \ldots, b_{n}\right)\right\}$, then the equation

$$
\sum_{j=1}^{n} a_{j} x b_{j}=y
$$

has a unique solution for all $y \in \mathcal{A}$. Moreover

$$
\|x\| \leq \frac{C}{\delta}\left(\frac{\max \{1, \delta\}}{\delta}\right)^{p}\|y\|
$$

where $p=s_{1}+\cdots+s_{n}+r_{1}+\cdots+r_{n}+c / 2+\varepsilon, \delta=\inf \left\{\lambda_{1} \mu_{1}+\cdots+\right.$ $\left.\lambda_{n} \mu_{n} \mid\left(\lambda_{1}, \ldots, \lambda_{n}\right) \in \sigma\left(a_{1}, \ldots, a_{n}\right),\left(\mu_{1}, \ldots, \mu_{n}\right) \in \sigma\left(b_{1}, \ldots, b_{n}\right)\right\}$, and $c$ is the balanced Hausdorff dimension of the set $\sigma\left(a_{1}, \ldots, a_{n}\right) \times$ $\sigma\left(b_{1}, \ldots, b_{n}\right)$.

(b) If , in addition, $s_{j}=r_{j}=0$ and $K$ is contained in an affine subspace of $\mathbb{R}^{2 n}$, then $\varepsilon$ in (a) can be omitted. In other words, $p=c / 2$.

Proof. The existence of the unique solution follows easily from Gel'fand theory. Indeed,

$$
\begin{aligned}
\sigma(\Lambda) & =\sigma\left(L_{a_{1}} R_{b_{1}}+\cdots+L_{a_{n}} R_{b_{n}}\right) \\
& \subseteq \sigma\left(L_{a_{1}}\right) \sigma\left(R_{b_{1}}\right)+\cdots+\sigma\left(L_{a_{n}}\right) \sigma\left(R_{b_{n}}\right) \\
& =\sigma\left(a_{1}\right) \sigma\left(b_{1}\right)+\cdots+\sigma\left(a_{n}\right) \sigma\left(b_{n}\right)=D .
\end{aligned}
$$

The proof of (8) was derived in [14], but for the convenience of the reader we shall outline it.

By Theorem 3.1, $\Lambda$ is a generalized scalar operator on a Banach space $\mathcal{A}$. Moreover $\left\|e^{i t \Lambda}\right\| \leq M\left(1+|t|^{p}\right)$, where $p=s+r+c / 2+\varepsilon$ in part (a) and $p=c / 2$ in part (b). From Theorem 2.1, it follows that

$$
f(\Lambda)=\frac{1}{\sqrt{2 \pi}} \int_{-\infty}^{\infty} \widehat{f}(\xi) e^{i \xi \Lambda} d \xi,
$$

where $\widehat{f}$ is the Fourier transform of $f$. Further, we can choose a function $f_{1} \in \check{L}_{1}^{p}$ equal to $1 / x$ in a neighborhood of $\{x \in \mathbb{R}|| x \mid \geq 1\}$. Set $f_{\delta}(x)=$ $f_{1}(x / \delta) / \delta$. Obviously, $f_{\delta}(x)=1 / x$ in a neighborhood of $\{x \in \mathbb{R}|| x \mid \geq \delta\} \supseteq$ $D \supseteq \sigma(\Lambda)$ for $\delta=\inf \left\{\lambda_{1} \mu_{1}+\cdots+\lambda_{n} \mu_{n} \mid \lambda_{j} \in \sigma\left(a_{j}\right), \mu_{j} \in \sigma\left(b_{j}\right)\right\}>0$, since $D$ does not contain 0 . Hence we have

$$
\begin{aligned}
\left\|\Lambda^{-1}\right\| & =\left\|f_{\delta}(\Lambda)\right\| \leq \frac{1}{\sqrt{2 \pi}} \int_{-\infty}^{\infty}\left|\widehat{f}_{\delta}(\xi)\right|\left\|e^{i \xi \Lambda}\right\| d \xi \\
& \leq \frac{M}{\sqrt{2 \pi}} \int_{-\infty}^{\infty}\left|\widehat{f}_{\delta}(\xi)\right|\left(1+|\xi|^{p}\right) d \xi .
\end{aligned}
$$

By a change of variables we see that $\widehat{f}_{\delta}(\xi)=\widehat{f}_{1}(\delta \xi)$, and thus 


$$
\begin{aligned}
\left\|\Lambda^{-1}\right\| & \leq \frac{M}{\sqrt{2 \pi}} \int_{-\infty}^{\infty}\left|\widehat{f}_{1}(\delta \xi)\right|\left(1+|\xi|^{p}\right) d \xi \\
& =\frac{M}{\sqrt{2 \pi} \delta^{p+1}} \int_{-\infty}^{\infty}\left|\widehat{f}_{1}(w)\right|\left(\delta^{p}+|w|^{p}\right) d w
\end{aligned}
$$

by substituting $\delta \xi=w$. The observation $\delta^{p}+|w|^{p} \leq(\max \{1, \delta\})^{p}\left(1+|w|^{p}\right)$ enables us to end the proof. The constant $C$ can be calculated as $C=$ $M \cdot \inf \left\|\left(1+|w|^{p}\right) \widehat{f}(w)\right\|_{L^{1}(\mathbb{R})}$, where the infimum is taken over all functions $f \in \check{L}_{1}^{s}$ which are equal to $1 / x$ in a neighborhood of $\{x|| x \mid \geq 1\}$. For the existence of such functions see [14] and references therein.

REMARK 3.5. In [16] the authors gave another estimate of $\|x\|$ in terms of $\|y\|$, using $d\left(a_{j}, b_{j}\right)=\inf \left\{|\langle u, v\rangle| /|u||v| \mid u \in \sigma\left(a_{j}\right), v \in \sigma\left(b_{j}\right)\right\}, d\left(a_{j}\right)=$ $\inf \left\{|u| \mid u \in \sigma\left(a_{j}\right)\right\}$ and $d\left(b_{j}\right)$. This estimate is

$$
\|x\| \leq C \max \left\{1, d\left(a_{j}\right)^{-s} d\left(b_{j}\right)^{-r}\right\} \frac{1+\left|\log d\left(a_{j}, b_{j}\right)\right|}{d\left(a_{j}\right) d\left(b_{j}\right) d\left(a_{j}, b_{j}\right)^{2 n+s+r}} .
$$

The estimates (8) and (9) are incomparable. Namely, if $n=1$, then $d\left(a_{j}, b_{j}\right)$ $=1, \delta=d\left(a_{j}\right) d\left(b_{j}\right)$, and (9) is sharper than (8). On the other hand, if $n=2$, $\sigma\left(a_{j}\right)=(t, 0), 1 \leq t \leq 2, \sigma\left(b_{j}\right)=(t \cos \varphi, t \sin \varphi), 1 \leq t \leq 2$, with $\varphi$ fixed, then $d\left(a_{j}\right)=d\left(b_{j}\right)=1, \delta=d\left(a_{j}, b_{j}\right)=\cos \varphi$, and (8) is sharper than (9) for $\varphi$ close to $\pi / 2$.

\section{Questions}

1. We believe that the additional condition on the set $K=\sigma\left(a_{1}, \ldots, a_{n}\right)$ $\times \sigma\left(b_{1}, \ldots, b_{n}\right)$ to have balanced Hausdorff dimension $c$ is superfluous, i.e. the usual notion of Hausdorff dimension is sufficient. However, we have no proof.

2. One can try to avoid the nondegeneracy condition in Theorem 2.5, by considering $Q_{\varepsilon}(x)=Q(x)+\varepsilon \sum_{j=1}^{2 n} x_{j}^{2}$.

3 . We are convinced that the technique applied in the proof of Theorem 2.5 can be upgraded in order to relax the condition on $K$. Namely we believe that, instead of assuming that $K$ is contained in a linear subspace of $\mathbb{R}^{2 n}$, it is enough to assume that it lies in a $c$-dimensional $C^{m}$ manifold for suitable $m$.

4. A more general frame for these investigations is $\Lambda(x)=\sum a_{j} x b_{j}$, $a_{j} \in \mathcal{A}, b_{j} \in \mathcal{B}$, and $x \in \mathcal{X}$; here $\mathcal{A}$ and $\mathcal{B}$ are unital Banach algebras, and $\mathcal{X}$ is a Banach $\mathcal{A}$ - $\mathcal{B}$-bimodule. The only problem with this general situation is to determine the relationship between the joint spectrum $\sigma\left(L_{a_{j}}, R_{b_{j}}\right)$ and the joint spectra $\sigma\left(a_{j}\right)$ and $\sigma\left(b_{j}\right)$. Note that $a_{j}, b_{j}$ are elements of a unital Banach algebra, and $L_{a_{j}}$ and $R_{b_{j}}$ are left and right multiplications on the bimodule $\mathcal{X}$. We believe that again a result analogous to that of Harte and Hernandez holds. 
5. In [20], Shul'man derived an ascent estimate for an elementary operator $\Lambda: B(H) \rightarrow B(H), \Lambda(X)=\sum_{j=1}^{n} A_{j} X B_{j}$, where $A_{j}$ and $B_{j}$ are commuting $n$-tuples of normal operators acting on a Hilbert space $H$. He proved that $\operatorname{asc}(\Lambda) \leq n-1$ and $\operatorname{asc}(\Lambda) \leq(c / 2]$, where $c$ is the Hausdorff dimension of the joint spectrum $\sigma_{\mathrm{T}}\left(A_{1}, \ldots, A_{n}\right)$. Here $(c / 2]$ denotes the least integer greater than or equal to $c / 2$, i.e. $(c / 2]=[c / 2]+1$ for noninteger $c / 2$, and $(c / 2]=c / 2$ for integer $c / 2$. (The number $c$ does not depend on the $n$-tuple $\left\{B_{n}\right\}$ !)

Our last question is whether an analogous result holds for pre-normal elements of a unital Banach algebra.

Acknowledgements. The authors are grateful to Professor R. Harte for his generous help in finding relevant literature and to the referee for useful comments, especially those related to Theorem 3.6.

\section{References}

[1] E. Albrecht, Funktionalkalküle in mehreren Veränderlichen für stetige lineare Operatoren auf Banachräumen, Manuscripta Math. 14 (1974). 1-40.

[2] C. A. Berenstein and R. Gay, Complex Variables: An Introduction, Grad. Texts in Math. 125, Springer, 1991.

[3] R. Bhatia and P. Rosenthal, How and why to solve the operator equation $A X-X B$ $=Y$, Bull. London Math. Soc. 29 (1997), 1-21.

[4] M. Sh. Birman and M. Z. Solomyak, Estimates for singular numbers of integral operators, Uspekhi Mat. Nauk 32 (1977), no. 1, 17-84 (in Russian).

[5] F. F. Bonsall and J. Duncan, Numerical Ranges of Operators on Normed Spaces and of Elements of Normed Algebras, London Math. Soc. Lecture Note Ser. 2, Cambridge Univ. Press, 1971.

[6] I. Colojoară and C. Foiaş, Theory of Generalized Spectral Operators, Gordon \& Breach, New York, 1968.

[7] E. A. Gorin and M. I. Karahanyan, An asymptotic variant of the Fuglede-Putnam theorem on commutators for Banach algebra elements, Mat. Zametki 22 (1977), no. 2, 179-188 (in Russian).

[8] R. Harte and C. Hernandez, On the Taylor spectrum of left-right multipliers, Proc. Amer. Math. Soc. 126 (1998), 397-404.

[9] L. Hörmander, The Analysis of Linear Partial Differential Operators I, Grundlehren Math. Wiss. 259, Springer, 1983.

[10] S. Lang, Algebra, Addison-Wesley, Reading, MA, 1965.

[11] G. Lumer and M. Rosenblum, Linear operator equations, Proc. Amer. Math. Soc. 10 (1959), 32-41.

[12] M. Mathieu, Elementary operators on prime $C^{*}$-algebras I, Math. Ann. 284 (1989), $223-244$.

[13] A. McIntosh and A. Pryde, A functional calculus for several commuting operators, Indiana Univ. Math. J. 36 (1987), 421-439.

[14] A. McIntosh, A. Pryde and W. Ricker, Estimates for solutions of the operator equation $\sum_{j=1}^{m} A_{j} Q B_{j}=U$, in: Special Classes of Linear Operators and Other Topics (Bucureşti, 1986), Oper. Theory Adv. Appl. 28, Birkhäuser, 1988, 197-207. 
[15] A. McIntosh, A. Pryde and W. Ricker, Systems of operator equations and perturbation of spectral subspaces of commuting operators, Michigan Math. J. 35 (1988), $43-65$.

[16] - - - - Comparison of joint spectra for certain classes of commuting operators, Studia Math. 88 (1988), 23-36.

[17] E. Saksman and H. O. Tylli, Weak essential spectra of multiplication operators on spaces of bounded linear operators, Math. Ann. 299 (1994), 299-309.

[18] - - - The Apostol-Fialkow formula for elementary operators on Banach spaces, J. Funct. Anal. 161 (1999), 1-26.

[19] V. S. Shul'man, Linear operator equations with generalized scalar coefficients, Dokl. Akad. Nauk SSSR 225 (1975), 56-58 (in Russian).

[20] —, Multiplying operators and spectral synthesis, ibid. 313 (1990), 1047-1051 (in Russian).

Matematički Fakultet

Univerzitet u Beogradu

Studentski trg 16-18

11000 Beograd, Serbia and Montenegro

E-mail: arsenovic@matf.bg.ac.yu

keckic@matf.bg.ac.yu

Received October 31, 2003

Revised version November 3, 2005 